\title{
Astragaloside IV Extends Lifespan of Caenorhabditis elegans by Improving Age-Related Functional Declines and Triggering Antioxidant Responses
}

\author{
Jianqin Zhang, ${ }^{1, *}$ Xiaoli Xue, ${ }^{1, *}$ Yuqi Qiao, ${ }^{1}$ Daqi Li, ${ }^{2}$ Qing Wei, ${ }^{3}$ Fusheng Zhang, ${ }^{1}$ and Xuemei Qin ${ }^{1}$
}

\begin{abstract}
Astragaloside IV (AS-IV) is a representative component of astragaloside saponins in dried roots of Astragali Radix. Astragaloside possesses a broad spectrum of pharmacological activities, including antibacterial, antifibrosis, antioxidant, anti-inflammatory, and neuroprotective effects. However, the role of AS-IV in antiaging remains unclear. In this article, we studied the function of AS-IV in antiaging by using the Caenorhabditis elegans (C. elegans) model. We showed that AS-IV can prolong the lifespan of $C$. elegans in a natural aging model, a paraquat injury model, and a heat stress model and improve the movement capacity of nematodes. ${ }^{1} \mathrm{H}-\mathrm{NMR}$ data indicate an improvement of glutamate content and a decrease in glucose in the AS-IV treatment group compared with the control. Further investigation revealed that AS-IV can induce the mRNA expression of superoxide dismutase (SOD) and catalase (CAT) genes and increase the activities of SOD and CAT in the nematode. Interestingly, AS-IV could not extend the lifespan of sod-1, sod-2, sod-3, sod-4, sod-5, ctl-1, ctl-2, $c t l-3$, and daf-16 mutants. These data indicate that AS-IV prevents aging via mainly improving age-related functional declines, the antioxidant capacity of nematodes and partially modulating the insulin/insulin growth factor 1 signaling pathway activity. Our results provide new insights into how AS-IV prevents and treats aging.
\end{abstract}

Keywords: astragaloside IV, Caenorhabditis elegans, antiaging, metabolomics, antioxidation

\section{Introduction}

I $\mathrm{N}$ MODERN SOCIETY, due to lifestyle changes and an aging population, diseases, including cardiovascular, diabetes, neurodegenerative disorders, and cancer, have been considered main dangers for human health. Recent data from epidemiologic studies show that public health costs are largely attributed to age-related disorders. ${ }^{1}$ Indeed, as the population ages, the medical burden will increase dramatically. ${ }^{2}$ A lot of research is now looking for the causes of aging and for developing new strategies to treat it, including medicinal and nonpharmacological methods such as physical exercise and low-calorie diet intake. ${ }^{1}$ Aging is a natural biological phenomenon that is prone to disease, but there are no drugs to delay human aging. ${ }^{3}$ Thus, currently, most research is focused on developing antiaging drugs. However, one of the most important challenges in aging research is how to find active compounds that delay life and clarify their underlying mechanism. Research reports that drugs and genetic interventions that can extend life can slow disease progression and improve health to some extent. ${ }^{4,5}$ The main pathways associated with aging-associated conditions are insulin/insulin growth factor 1 signaling (IIS), the mammalian (or mechanistic) target of rapamycin, and sirtuin pathways. Most works demonstrated that insulin/insulin-like signaling, ${ }^{6}$ mitochondrial respiration pathways, ${ }^{7}$ and dietary restriction ${ }^{8}$ affected the development of aging-related diseases. ${ }^{9,10}$ Therefore, the constituent factors of these conservative aging pathways can be new targets that can be operated to provide protection from aging-related diseases. ${ }^{11}$

Astragaloside IV (AS-IV), a lanolin alcohol-shaped tetracyclic triterpenoid saponin, is derived from the dried root of Astragalus membranaceus (Supplementary Fig. S1). Decoctions prepared from the dried root, also known as

\footnotetext{
${ }^{1}$ Modern Research Center for Traditional Chinese Medicine, Shanxi University, Taiyuan, China.

${ }^{2}$ Institute of Plant Protection, Shanxi Academy of Agricultural Sciences, Taiyuan, China.

${ }^{3}$ Center for Reproduction and Health Development, Institute of Biomedicine and Biotechnology, Shenzhen Institutes of Advanced Technology, Chinese Academy of Sciences (CAS), Shenzhen, China.

*These authors contributed equally to this work.
} 
Astragali Radix or "Huangqi," are widely used in traditional Chinese medicine for the treatment of inflammation, viral and bacterial infections, and even cancer. Astragali Radix has the function of scavenging free radical, antioxidation, inhibition of inflammation, immune regulation, and cardiovascular protection. ${ }^{12}$ A major compound detected in Astragali Radix products is AS-IV. Studies using various cellular and animal models have shown that AS-IV has anti-inflammatory activity ${ }^{13}$ and antioxidative stress activity. ${ }^{14}$ However, little is known about whether AS-IV has antiaging activity.

From single-cell eukaryotes to mammals, the signaling pathways of aging are basically the same. ${ }^{9} C$. elegans is a good model for screening drugs with delaying life effects, which is attributed to the excellent merits of a short lifespan and easy for genetic manipulation. Metabolomics is a powerful technique for systematically analyzing changes in endogenous metabolites during life. ${ }^{15}$ Because nuclear magnetic resonance (NMR) owns many distinguishing features, such as rapidity, great stability, and nonselectivity. NMR has been an extensively applied method in metabolomics analysis. ${ }^{16}$ This technique may be particularly effective when the effects and mechanisms of drugs on organisms are unknown.

In this study, the effects and mechanisms of AS-IV against aging were investigated. We found that AS-IV could extend the lifespan of the nematode $C$. elegans and improve stress resistance. Subsequent genetic analyses with a series of worm mutants revealed that AS-IV extends the lifespan of $C$. elegans through improving age-related functional declines and triggering antioxidant responses.

\section{Materials and Methods}

\section{Strains}

C. elegans strains wild-type N2 (Bristol), sod-1 (tm776), sod-2 (gk257), sod-3 (tm760), sod-4 (gk101), sod-5 (tm1146), ctl-1 (ok1242), ctl-2 (ok1137), ctl-3 (ok2042), and Escherichia coli strain OP50 were provided by Professor Enbo Ma (Research Institute of Applied Biology in Shanxi University, Shanxi, China). C. elegans strains sir-2.1 (ok434) and daf-16 (mu86) were purchased from the Caenorhabditis Genetics Center (CGC).

Strain maintenance was performed at $20^{\circ} \mathrm{C}$ on nematode growth medium (NGM) agar plates containing a lawn of E. coli OP50 as the food source as described elsewhere. ${ }^{3}$

\section{Chemicals}

AS-IV (Jiangsu Yongjian Pharmaceutical Technobgy Co., Ltd., China) was dissolved in DMSO for storage. Then, the compounds were diluted in PBS to a series of concentrations before use. For the AS-IV treatment group, the compounds were added in the NGM plates, and the final DMSO concentration was $0.1 \%$. The negative control group contained the same concentration of DMSO $(0.1 \%)$ as the AS-IV treatment group. FudR (5-fluoro-2'-deoxyuridine; Sigma) was added to the NGM plates in each experiment to prevent selffertilization.

\section{AS-IV biological assays}

Worm eggs were incubated in the $\mathrm{M} 9$ buffer $\left(3 \mathrm{~g} \mathrm{KH}_{2} \mathrm{PO}_{4}\right.$, $6 \mathrm{~g} \mathrm{Na}_{2} \mathrm{HPO}_{4}, 5 \mathrm{~g} \mathrm{NaCl}$ in $1 \mathrm{~L}$ of $\mathrm{ddH}_{2} \mathrm{O}$ ) overnight at $20^{\circ} \mathrm{C}$. The Eppendorf tube containing L1 larvae was put on ice for
15 minutes, and it was centrifuged at $3000 \mathrm{r} / \mathrm{min}$; the synchronized L1 larvae were cultured on an NGM plate with OP50. Then, late L4 larvae were cultured on NGM plates containing inactivated $E$. coli $\mathrm{OP} 50\left(65^{\circ} \mathrm{C}, 30\right.$ minutes $)$ and $100 \mu \mathrm{M}$ of FudR (Sigma) at $20^{\circ} \mathrm{C}^{17}$ Experimental day 0 was defined as the day when L4 larvae were transferred to an NGM plate with or without AS-IV. The number of alive nematodes was counted each day. Worms that were unresponsive to stimulus were set as dead. ${ }^{8}$ The lifespan assay was repeated for three biological replications in each group. The total number of nematodes in each group is 120 .

For the thermo-tolerance assay, $C$. elegans at day 5 were cultured on NGM plates at $35^{\circ} \mathrm{C}$ in an incubator. The number of alive nematodes was scored every 2 hours. ${ }^{11} \mathrm{Six}$ plates were used in each group. Totally, 120 worms were used in each group.

For the oxidative stress assay, worms were cultured on NGM plates, including $5 \mathrm{mM}$ of paraquat (Sigma). Six plates were used in each group. The surviving animals were scored every other day. This experiment was conducted for six biological replicates. Totally, 120 nematodes were used in each group.

Body bend was monitored by computer-tracking techniques. ${ }^{18}$ The L4 larvae were cultured on NGM plates, including $5 \mu \mathrm{M}$ AS-IV, and control NGM plates lacking AS-IV. The nematodes were transferred to NGM medium without OP50 and moved for 30 minutes. Afterward, the number of bends of the body within 1 minute was recorded as the body bending frequency index. The experiment was repeated for 10 biological replications.

\section{NMR measurements}

One hundred twenty synchronized young adult worms were transferred to 10 plates with or without $5 \mu \mathrm{M}$ of AS-IV. In this experiment, we set three groups: (1) a control group: Synchronized young adult worms were cultured in NGM plates without $5 \mu \mathrm{M}$ of AS-IV for 3 days; three-day-old worms were collected as control samples. (2) an aging group: Synchronized young adult worms were cultured in NGM plates without $5 \mu \mathrm{M}$ of AS-IV for 8 days; eight-day-old worms were collected as aging samples. (3) an AS-IV group: Synchronized young adult worms were cultured in NGM plates with $5 \mu \mathrm{M}$ of AS-IV for 8 days; the eight-day-old worms treated by $5 \mu \mathrm{M}$ of AS-IV were set as AS-IV group samples. The worms were collected in $1.5 \mathrm{~mL}$ new tubes. The samples were centrifuged at $12,000 \mathrm{rpm}$ for $5 \mathrm{~min}-$ utes; the precipitated residues were used as samples, which were stored at $-80^{\circ} \mathrm{C}$ until extraction.

Nuclear magnetic sample preparation method followed a protocol described by Geier FM, ${ }^{19}$ with the following modifications: About $600 \mathrm{mg}$ of nematodes was extracted by using $2.4 \mathrm{~mL}$ of $80 \%$ methanol with ultrasonic crushing for 15 minutes under ice. The mixture was centrifuged, and the supernatants were evaporated to dryness under vacuum. The NMR samples were prepared by dissolving $10-20 \mathrm{mg}$ portions of metabolite extract in $0.6 \mathrm{~mL}$ of $10 \%$ $\mathrm{D}_{2} \mathrm{O}$ (Qingdao Tenglong Weibo Technology Co., Ltd.). The samples were centrifuged at $13,000 \mathrm{rpm}$ for 10 minutes. The supernatant was put into $5 \mathrm{~mm}$ NMR tubes. Samples were analyzed by using a Bruker $600 \mathrm{MHz}$ Avance III NMR spectrometer. 
All ${ }^{1} \mathrm{H}$-NMR spectra were corrected with MestReNova software (version 8.0.1, Spain). Trimethylsilylpropanoic acid was used as a spectral reference for all samples. A linear interpolation method was used to align the spectra. Water signals were removed before data normalization. All spectra were segmented at $0.01 \mathrm{ppm}$ intervals across $\delta 0.80-9.00$ for C. elegans tissue extracts. Simca-P 13.0 software (Umetrics, Sweden) was used to conduct multivariate data analysis. Principal component analysis (PCA), Partial least-squares discriminant analysis (PLS-DA), and Orthogonal-projection to latent structure-discriminate analysis (OPLS-DA) were applied to analyze the metabolic profiles between groups and identify metabolites. The validity of the models was assessed by 200 permutation tests and the CV-ANOVA method.

\section{Mutant assay}

The details of this experiment are the same as for the lifespan assay. For the mutant assay, 20 worms were used for each replicate. The lifespan assay for each mutant included six biological replicates. Specific C. elegans mutants used were: sod-1 (tm776), sod-2 (gk257), sod-3 (tm760), sod-4 (gk101), sod-5 (tm1146), ctl-1 (ok1242), ctl-2 (ok1137), ctl-3 (ok2042), sir-2.1 (ok434), and daf-16 (mu86).

\section{Enzyme assay}

About 2000 synchronized L4 larvae nematodes were divided into two groups and cultured for 8 days. The NGM plates containing $5 \mu \mathrm{M}$ of AS-IV were applied in the experimental group, whereas the NGM plates without AS-IV were used in the negative control group. The manufacturer's instructions of enzyme extraction were followed (Nanjing Jiancheng, China). The activities of SOD and CAT were tested by a Microplate reader. SOD activity was determined at $450 \mathrm{~nm}$ by using a xanthine and xanthine oxide system. CAT activity was measured by monitoring the amount of complex compound at $240 \mathrm{~nm}$ due to $\mathrm{H}_{2} \mathrm{O}_{2}$ decomposition. The detailed methods of this experiment are the same as previously described. ${ }^{20}$

\section{mRNA expression of genes}

For each experiment, about 1000 worms were used to extract total RNA in the same condition as described for enzyme assay. The cDNA Reverse Transcription M-MLV Kit (TaKaRa) was used to prepare the cDNA. The gene expression levels (sod-1, sod-2, sod-3, sod-4, sod-5, ctl-1, ctl-2, ctl-3, $j k k-1, j n k-1$, and $k g b-2)$ were determined in different groups by reverse transcription quantitative PCR (RT-qPCR) using SYBR Green Real-Time PCR Master Mixes according to the manufacturer's instructions (TOYOBO). RT-qPCR was performed with four biological replications on a LightCycler 480 instrument (Roche, Inc., Basel, Switzerland). $c d c-42$ was used as a reference gene. ${ }^{21}$ The primers used for RT-qPCR are listed in Supplementary Table S1. The mRNA expression of each gene was calculated according to $2^{-\Delta \Delta \mathrm{CT}}$ method. ${ }^{22}$

\section{Statistical analysis}

SPSS 16.0 software (SPSS, Inc., Chicago, IL) was used for statistical analysis. Differences between treatments were evaluated by using a Student's $t$-test. For lifespan analyses, Kaplan-Meier lifespan analysis was carried out by using the log-rank test. ${ }^{11} \mathrm{~A} p$-value of $<0.05$ was considered statistically significant. All of the results are expressed as the means \pm standard error of the mean (SEM).

\section{Results}

\section{AS-IV increases the lifespan of C. elegans}

To confirm the longevity manipulation properties of ASIV, N2 worms were treated with a serial concentration of AS-IV ranging from 0 to $10 \mu \mathrm{M}$. The results showed that $5 \mu \mathrm{M}$ of AS-IV could significantly extend the lifespan of C. elegans $(p<0.001)$ (Fig. 1 and Supplementary Fig. S2A). The mean, median, and maximum lifespan of worms increased by up to $27.8 \%, 35.7 \%$, and $16.7 \%$, respectively (Table 1 and Fig. 1). Bell-shaped curves appear in the analysis of lifespans' means and medians, indicating that there is a hormetic phenomenon in the effect of AS-IV on the body (Supplementary Fig. S2B and C).

\section{AS-IV increases $\mathrm{C}$. elegans resistance to stressful environments}

To test whether AS-IV could enhance the heat stress resistance of C. elegans, adult worms were exposed to $5 \mu \mathrm{M}$ of AS-IV and kept at $35^{\circ} \mathrm{C}$. The mean lifespan of worms cultured on NGM plates, including $5 \mu \mathrm{M}$ of AS-IV, was extended by up to $14.9 \%(p<0.05)$, indicating that AS-IV can significantly strengthen the heat stress resistance (Fig. 2A). Similarly, to evaluate whether AS-IV-treated worms were resistant to oxidative stress, worms were cultured on plates with $5 \mu \mathrm{M}$ of AS-IV and $5 \mathrm{mM}$ of paraquat at $20^{\circ} \mathrm{C}$. The lifespan of worms treated with $5 \mu \mathrm{M}$ of AS-IV was increased by at least $27.8 \%$. The survival percentage of worms in the AS-IV experimental group was extensively higher than that of control worms $(p<0.001)$, indicating that AS-IV can significantly improve the oxidative resistance of worms (Fig. 2B). These results suggest that AS-IV could prolong the health lifespan of worms and improve their heat and oxidative damage resistance.

\section{AS-IV improves the movement of $\mathrm{C}$. elegans}

To clarify whether AS-IV can improve the athletic ability of C. elegans, we tested the body bending frequency, which is an indicator of nematode movement behavior. The results showed that the movement capacity of the N2 nematode was improved significantly in the $5 \mu \mathrm{M}$ of the AS-IV group than in the control group (Fig. 2C, $* * p<0.001$ ).

\section{Metabolites identification by ${ }^{1} \mathrm{H}-\mathrm{NMR}$ spectra}

The typical ${ }^{1} \mathrm{H}-\mathrm{NMR}$ spectra of control $C$. elegans are shown in Supplementary Fig. S3. NMR signals were assigned to special metabolites based on the literature ${ }^{23}$ and further confirmed by NMR databases, such as the Human Metabolome Database and the Biological Magnetic Resonance Bank. The detected metabolites in C. elegans included two organic acids (taurine, acetate), five amino acids (leucine, glutamate, glutamine, asparagine, threonine), three tricarboxylic acid cycle (TCA) intermediates (fumarate, succinate, and citrate), and glucose. Endogenous metabolites identified in $C$. elegans are shown in Supplementary Table S2. 

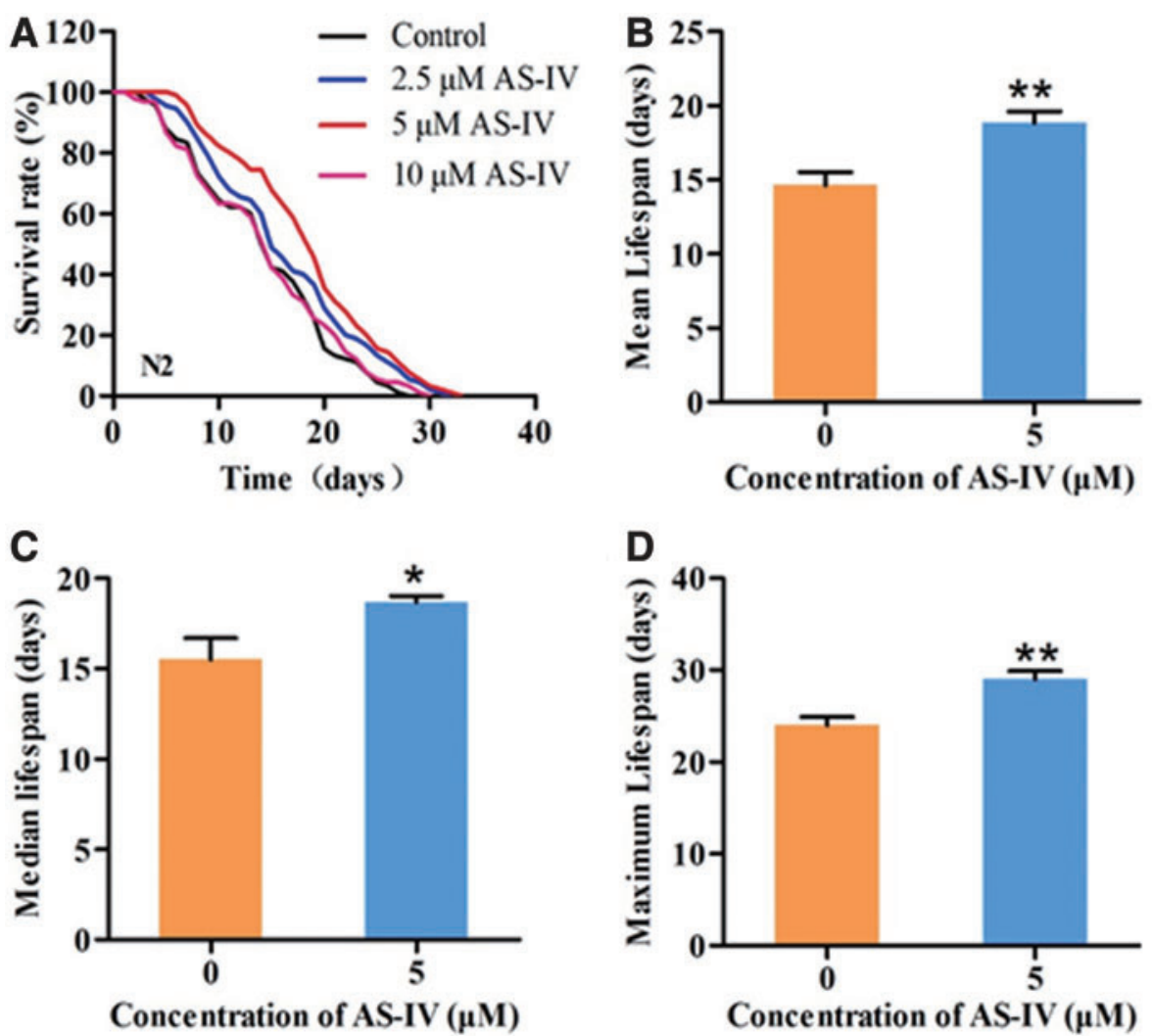

FIG. 1. Effects of AS-IV on the lifespan of Caenorhabditis elegans. (A) Lifespan curves of C. elegans exposed to different concentrations of AS-IV. Wild-type N2 worms raised at $20^{\circ} \mathrm{C}$ on NGM plates containing $2.5,5$ or $10 \mu \mathrm{M}$ of AS-IV. Survival curves were obtained by SPSS software. The Kaplan-Meier lifespan analysis was performed, and $p$-values were calculated by using log-rank test. (B) Effect of AS-IV on the mean lifespan of C. elegans. (C) Effect of AS-IV on the median lifespan of $C$. elegans. (D) Effect of AS-IV on the maximum lifespan of $C$. elegans. The Student's $t$-test was performed. The results are presented as the means \pm standard errors of three biological replications $(n=3)$. $* p<0.05$, $* * p<0.01$. AS-IV, Astragaloside IV; NGM, nematode growth medium. Color images are available online.

Visual inspection of ${ }^{1} \mathrm{H}-\mathrm{NMR}$ spectra of $C$. elegans revealed significant differences between aging and control groups. To detect more details about AS-IV induced metabolic changes, multivariate analysis was applied. In the PCA score plot (Fig. 3A) of the first two principal components (PC1: 60.0\%; PC2: 24.4\%), a clear separation can be observed between control, aging, and $5 \mu \mathrm{M}$ AS-IV groups. At the same time, the control group is closer to the $5 \mu \mathrm{M}$ of the AS-IV group, indicating that administration of $5 \mu \mathrm{M}$ AS-IV can delay aging of nematodes. Further, $\mathrm{R}^{2}$ and $\mathrm{Q}^{2}$ values were lower than the original ones, suggesting the validity of the discriminant model (Fig. 3B). The PLS-DA model was also constructed and validated by 200 permutation tests. The corresponding OPLS-DA was used to determine the poten- tial biomarkers contributing to the separation (Fig. 3C). The OPLS-DA loading plot $(p<0.05$, VIP $>1)$ was used to detect metabolites that facilitate separation (Fig. 3D). We find that the major metabolite variations in C. elegans after $5 \mu \mathrm{M}$ of AS-IV treatment are the decline of glucose, as well as the elevation of leucine, acetate, glutamate, glutamine, citrate, succinate, asparagine, taurine, threonine, and fumarate (Supplementary Fig. S4).

We applied the ingenuity network analysis to determine metabolic pathways affected by AS-IV. The potential biomarkers in $C$. elegans were analyzed by MetPA. Here, six metabolic pathways, including (1) TCA cycle; (2) sulfur metabolism; (3) alanine, aspartate, and glutamate metabolism; (4) valine, leucine, and isoleucine biosynthesis;

Table 1. Effects of Astragaloside IV on LifEsPan of CaENORHabditis ELEGANS

\begin{tabular}{lccccc}
\hline AS-IV/ $\mu M$ & Number & Mean/d & Median/d & $\mathrm{p}$ (log-rank test $)$ & Max/d \\
\hline 0 & 120 & $14.4 \pm 0.7$ & $14.0 \pm 0.6$ & - & $25.7 \pm 0.5$ \\
2.5 & 120 & $16.4 \pm 0.8$ & $15.0 \pm 0.8$ & $<0.05$ & $29.2 \pm 0.5$ \\
5 & 120 & $18.4 \pm 0.7$ & $19.0 \pm 0.7$ & $<0.001$ & $30.0 \pm 0.6$ \\
10 & 120 & $14.5 \pm 0.7$ & $15.0 \pm 0.6$ & $>0.05$ & $26.7 \pm 0.8$ \\
\hline
\end{tabular}

Mean lifespan was calculated from three biological replicates in each group. The data were analyzed by using Kaplan-Meier survival curves in SPSS packages (16.0). Differences between the data were considered significant at $p \leq 0.05$. 

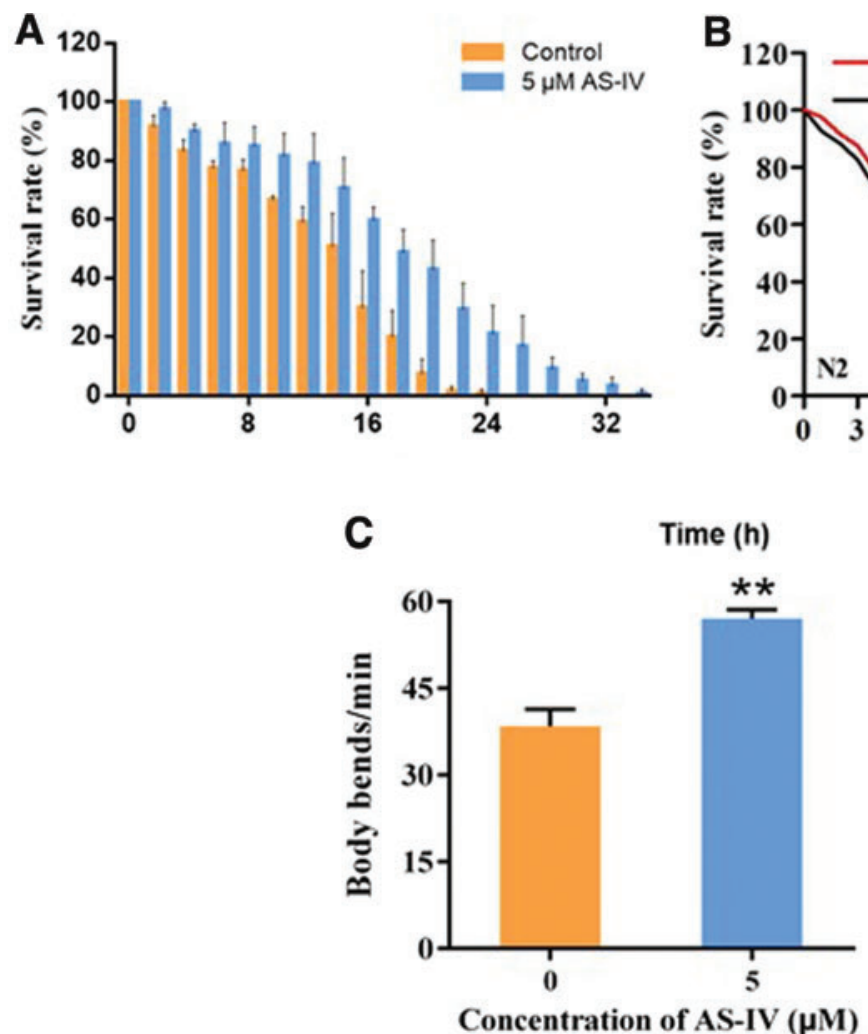

( $\mathrm{AS}-\mathrm{IV}(\mu \mathrm{M})$

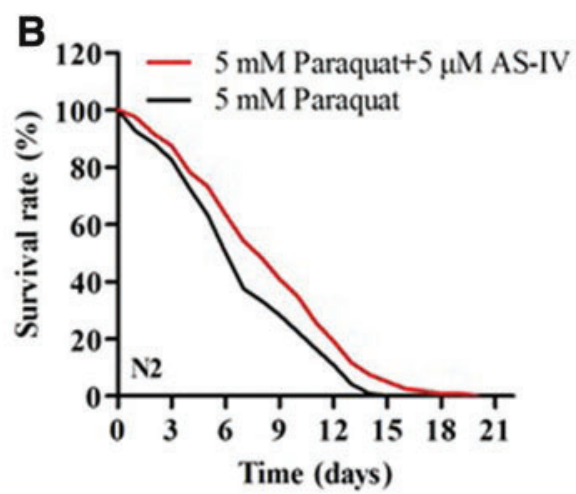

FIG. 2. Effects of AS-IV on the phenotype of $C$. elegans. (A) Percent survival of wild-type worms exposed to $5 \mu$ M of AS-IV at $35^{\circ} \mathrm{C}$. The control group was cultured on normal medium. Totally, 120 worms were used in each group. Each bar indicates the mean lifespan of three independent experiments with error bars $(p<0.0001, \log$-rank test). (B) Percent survival of wild-type worms cultured with $5 \mathrm{mM}$ of paraquat while exposed to $5 \mu \mathrm{M}$ of AS-IV ( $p<0.001, \log$-rank test). Totally, 120 nematodes were used in each group. (C) The age-related movements of worms treated with $5 \mu \mathrm{M}$ of AS-IV. The control group was cultured with normal medium $(* * p<0.001$, Student $t$-test). The experiment was repeated for 10 biological replications. Color images are available online.

(5) glyoxylate and dicarboxylate metabolism; and (6) taurine and hypotaurine metabolism, were established (Supplementary Fig. S5). These are likely to account for the host response to aging processes. The summary of pathway alterations after $5 \mu \mathrm{M}$ of AS-IV treatment is shown in Supplementary Figure S6.

\section{Effect of AS-IV on nematode sir-2.1 mutant}

The results of NMR data suggest that the antiaging effects of AS-IV may be related to energy metabolism (dietary restriction). To confirm this result, we examined the antiaging effects of AS-IV on sir-2.1 (ok434) mutants, which are commonly used as energy metabolism mutants. The results demonstrated that the survival rate of sir-2.1 mutants was significantly increased in the $5 \mu \mathrm{M}$ of the AS-IV group, as compared with the control group (Fig. 4), indicating that the mechanism of AS-IV on antiaging may not be through the energy metabolism pathway of sir-2.1.

\section{Effect of AS-IV on immunoregulatory genes}

The results of the NMR data suggest that the antiaging effects of AS-IV may be related to immunomodulatory effects. To investigate this hypothesis, we measured the mRNA expression levels of immunoregulatory genes. As shown in Figure 5, the transcription of $j k k-1, j n k-1$, and $k g b-2$ was not significantly different in the $5 \mu \mathrm{M}$ of the AS-IV group, compared with the control group.

\section{Effect of AS-IV on the antioxidant activity of nematodes}

The results of the NMR data suggest that the antiaging properties of AS-IV may be related to antioxidation. To investigate this hypothesis, we measured antioxidant enzyme activity, the mRNA expression level of antioxidant genes of nematodes, and the lifespan of mutants sod-1 (tm776), sod-2 (gk257), sod-3 (tm760), sod-4 (gk101), sod-5 (tm1146), ctl-1 (ok1242), ctl-2 (ok1137), ctl-3 (ok2042), and daf-16 (mu86). The results showed that $5 \mu \mathrm{M}$ of AS-IV significantly increased SOD and CAT activity, compared with the control group (Fig. 6A). As shown in Figure 6B, the transcription levels of $\operatorname{sod}-3$, sod-4, and sod-5 were significantly increased in the $5 \mu \mathrm{M}$ AS-IV group compared with the control group $(p<0.05)$. However, there were no statistically significant differences for sod-1 and sod-2. As shown in Figure 6C, the transcription levels of $c t l-2$ and $c t l$ 3 were significantly increased in the $5 \mu \mathrm{M}$ AS-IV group compared with the control group $(p<0.05)$. The expression of $c t l-1$ also increased, but there was no statistically significant difference. To further determine the involvement of antioxidation-related genes in regulating the lifespan of nematodes, we investigated the effect of $5 \mu \mathrm{M}$ AS-IV on the 

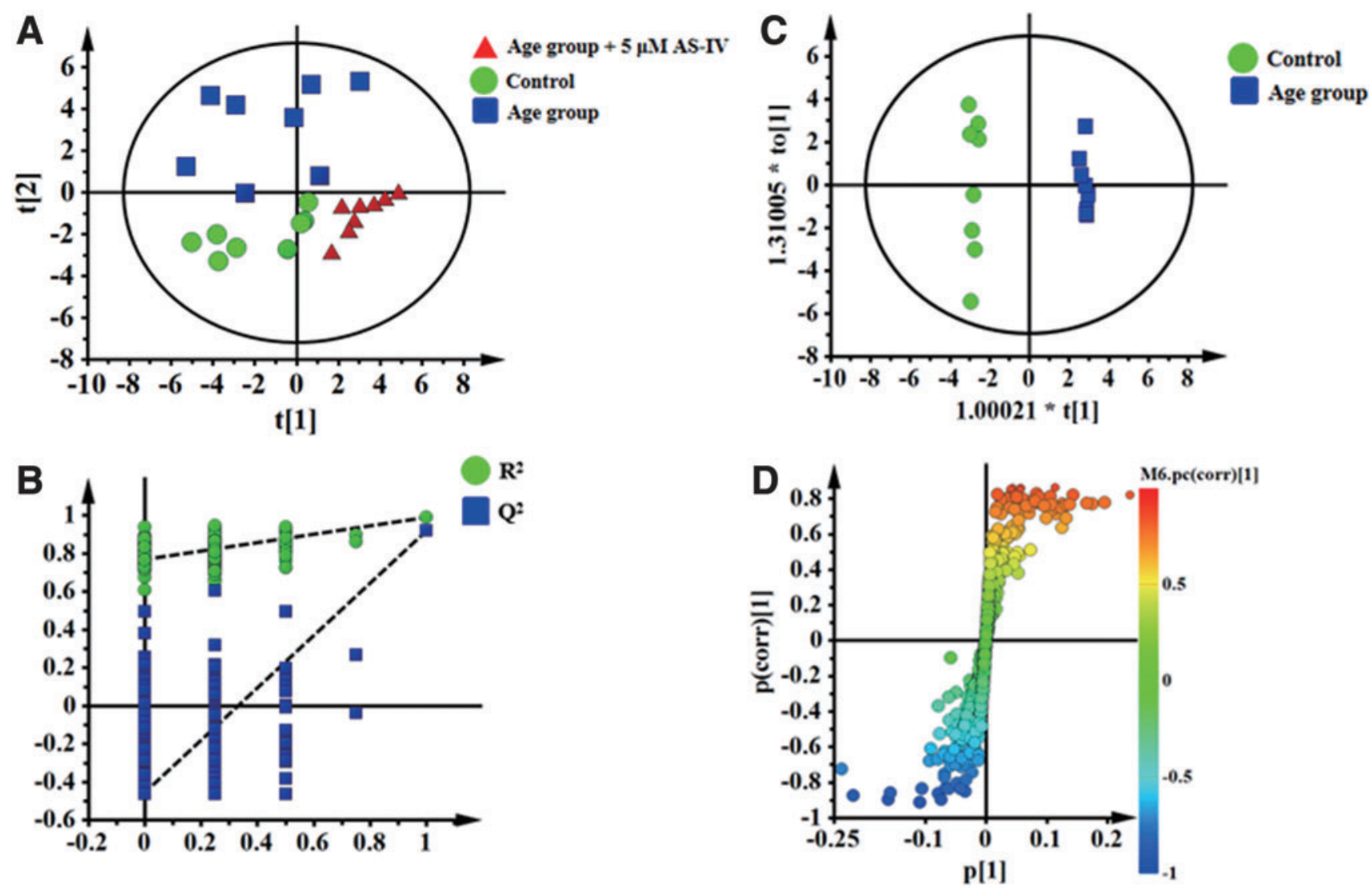

FIG. 3. Effects of AS-IV on C. elegans metabolic chemistry. (A) The principal component analysis score plot (PC1 vs. PC2) for C. elegans. (B) The orthogonal projections to latent structures discriminant analysis score plot (PC1 vs. PC2). (C) The $y$-axis shows the $\mathrm{R}^{2} \mathrm{Y}$ (green-filled dots) and $\mathrm{Q}^{2} \mathrm{Y}$ (blue-filled square) values of every model, whereas the $x$-axis indicates the correlation coefficient between original and permuted data response. The $\mathrm{Y}$ intercepts of plot for the $\mathrm{R}^{2} \mathrm{Y}$ and $\mathrm{Q}^{2} \mathrm{Y}$ in every model are expressed as numbers. (D) S-plot with color coefficient scale bar for the model discriminating the coat and kernel of mung bean, permutation test plots. In this work, we set three groups: (1) a control group consisting of synchronized young adult worms cultured in NGM plates without $5 \mu \mathrm{M}$ of AS-IV for 3 days, (2) an aging group consisting of synchronized young adult worms cultured in NGM plates without $5 \mu \mathrm{M}$ of AS-IV for 8 days, and (3) an AS-IV group consisting of synchronized young adult worms cultured in NGM plates with $5 \mu \mathrm{M}$ of AS-IV for 8 days. Color images are available online.

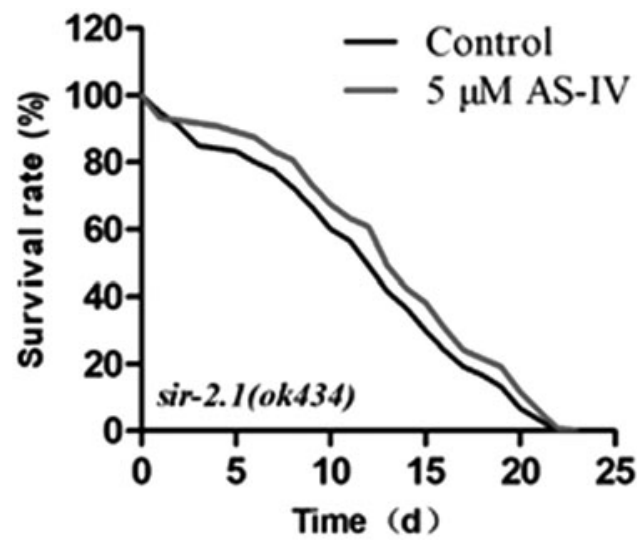

FIG. 4. Survival curves of sir-2.1 (OK434) C. elegans mutants treated with $5 \mu \mathrm{M}$ of AS-IV at $20^{\circ} \mathrm{C}$. Survival curves were obtained by GraphPad Prism software. The Kaplan-Meier lifespan analysis was performed, and $p$-values were calculated by using log-rank test. lifespan of sod and ctl nematode mutants. The results showed that the lifespan of sod (sod-1, sod-2, sod-3 sod-4, and sod-5) and ctl (ctl-1, ctl-2, and ctl-3) nematodes had no effect $(p \geq 0.05)$ after $5 \mu \mathrm{M}$ AS-IV exposure, when compared with the control (Fig. 7).

\section{Effect of AS-IV on nematode daf-16 mutant}

The results of NMR data showed that glucose content was decreased in C. elegans exposed to the $5 \mu \mathrm{M}$ of AS-IV. daf- 16 has been found to mediate glucose to trehalose interconvert, thus leading to lifespan extension. ${ }^{24}$ The FOXO transcription factor DAF-16 is required for IIS mutations to extend lifespan. ${ }^{25}$ Therefore, we checked whether daf- 16 is required for AS-IV to affect longevity. The results showed that AS-IV failed to extend the lifespan of mutant $d a f-16$ worms (Fig. 7).

\section{Discussion}

AS-IV is the main active ingredient isolated from Astragali Radix. AS-IV has various pharmacological activities, including antibacterial, antifibrotic, ${ }^{26}$ anti-inflammatory, ${ }^{13}$ 


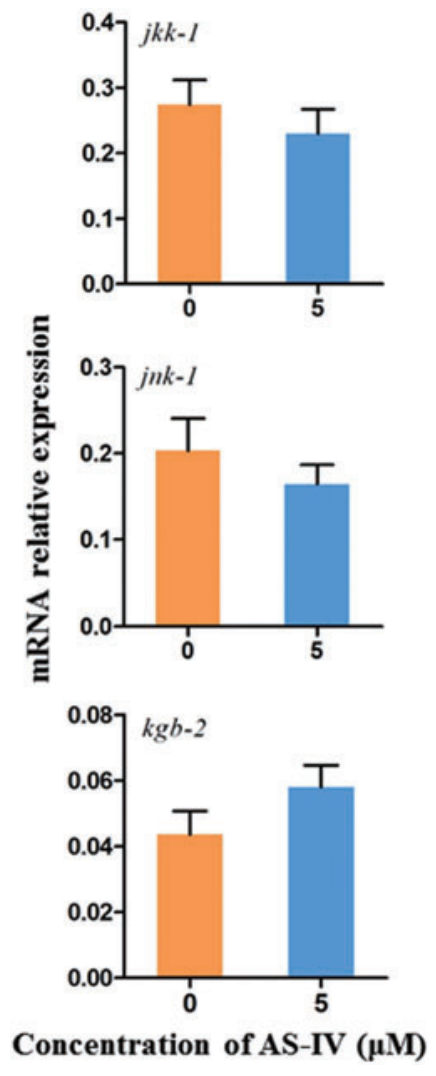

FIG. 5. Relative expression levels of $j k k-1, j n k-1$, and $k g b$ 2 genes in $C$. elegans after AS-IV treatment. The relative expression levels of target genes in different groups were evaluated by RT-qPCR. The $c d c-42$ gene was used as a reference. Results are presented as the means \pm standard errors of four biological replications $(n=4)$. The data were analyzed by GraphPad Prism software. RT-qPCR, reverse transcription quantitative PCR. Color images are available online.

antioxidant, ${ }^{14}$ and neuroprotective effects. ${ }^{27}$ There is growing evidence to suggest that anti-inflammatory, ${ }^{28}$ antioxidant,$^{29}$ and other mechanisms ${ }^{30}$ can delay aging. Therefore, we hypothesized that the AS-IV can delay the aging of C. elegans through anti-inflammatory and antioxidation. Our work shows that AS-IV extends the lifespan of $C$. elegans through improving age-related functional declines and triggering antioxidant responses.

Generally, C. elegans expanding lifespan through pharmacological intervention or genetic mutation often owns strong stress resistance to high temperature and reactive oxygen species (ROS) ${ }^{11}$ Stress resistance can be set as a longevity biomarker. ${ }^{31-33}$ In our work, AS-IV significantly improves the health lifespan of $C$. elegans $(p<0.001)$ and increases the heat and oxidative damage resistance.

In this study, the antiaging mechanism of AS-IV was explored by using nuclear magnetic metabolomics $\left({ }^{1} \mathrm{H}-\mathrm{NMR}\right)$ and molecular biology. ${ }^{1} \mathrm{H}-\mathrm{NMR}$ showed that the nematode aging group and control group can be significantly distinguished, indicating that the nematode metabolic profile changes due to aging. Our results showed that AS-IV plays a certain role in regulating metabolic markers of nematodes. According to the changes mentioned earlier in differential metabolites, the antiaging effect of AS-IV can be discussed as follows:
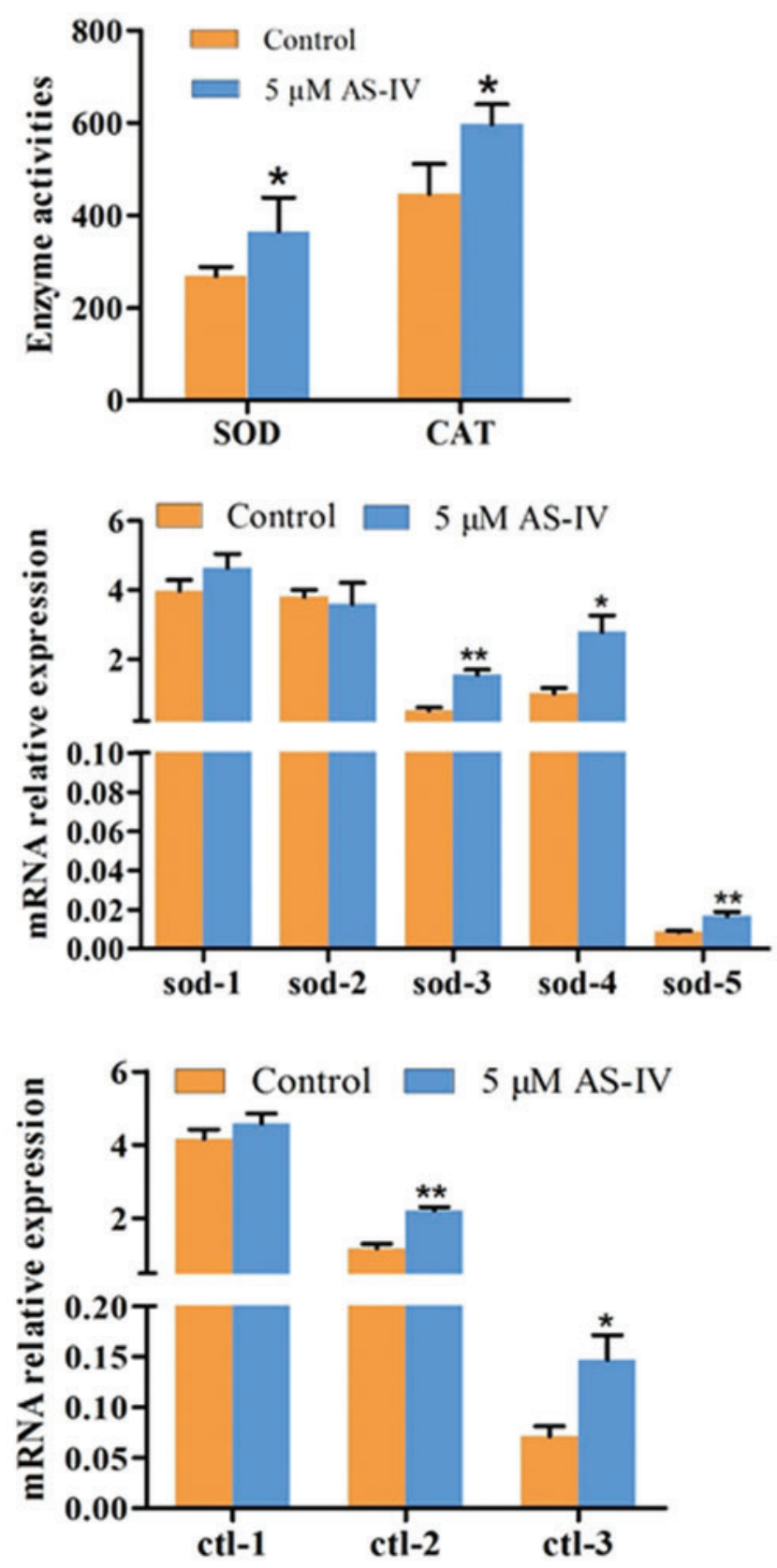

FIG. 6. Effect of AS-IV on C. elegans SOD and CAT activity. (A) Antioxidant enzyme activity when exposed to different concentrations of AS-IV. SOD units $=\mathrm{U}$ mg-1 protein, CAT units $=$ mmol min-1 mg-1 protein. (B) Relative expression levels of sod as evaluated by RT-qPCR. (C) The relative expression levels of $C t l$ as evaluated by RT-qPCR. The mRNA level in each group was normalized by cdc-42 as a reference gene. Results are means \pm standard error of four biological replications $(n=4)$. The date were analyzed by Graphpad Prism software. ${ }^{*} p<0.05,{ }^{*} p<0.01$, calculated by two-tailed $t$-test. Color images are available online.

(1) The leucine in the AS-IV group had significant reversal compared with the aging group, indicating that the antiaging effect of AS-IV may be related to energy metabolism. As the ability to absorb and degrade glucose in organisms declined, the glucose content increased during the 

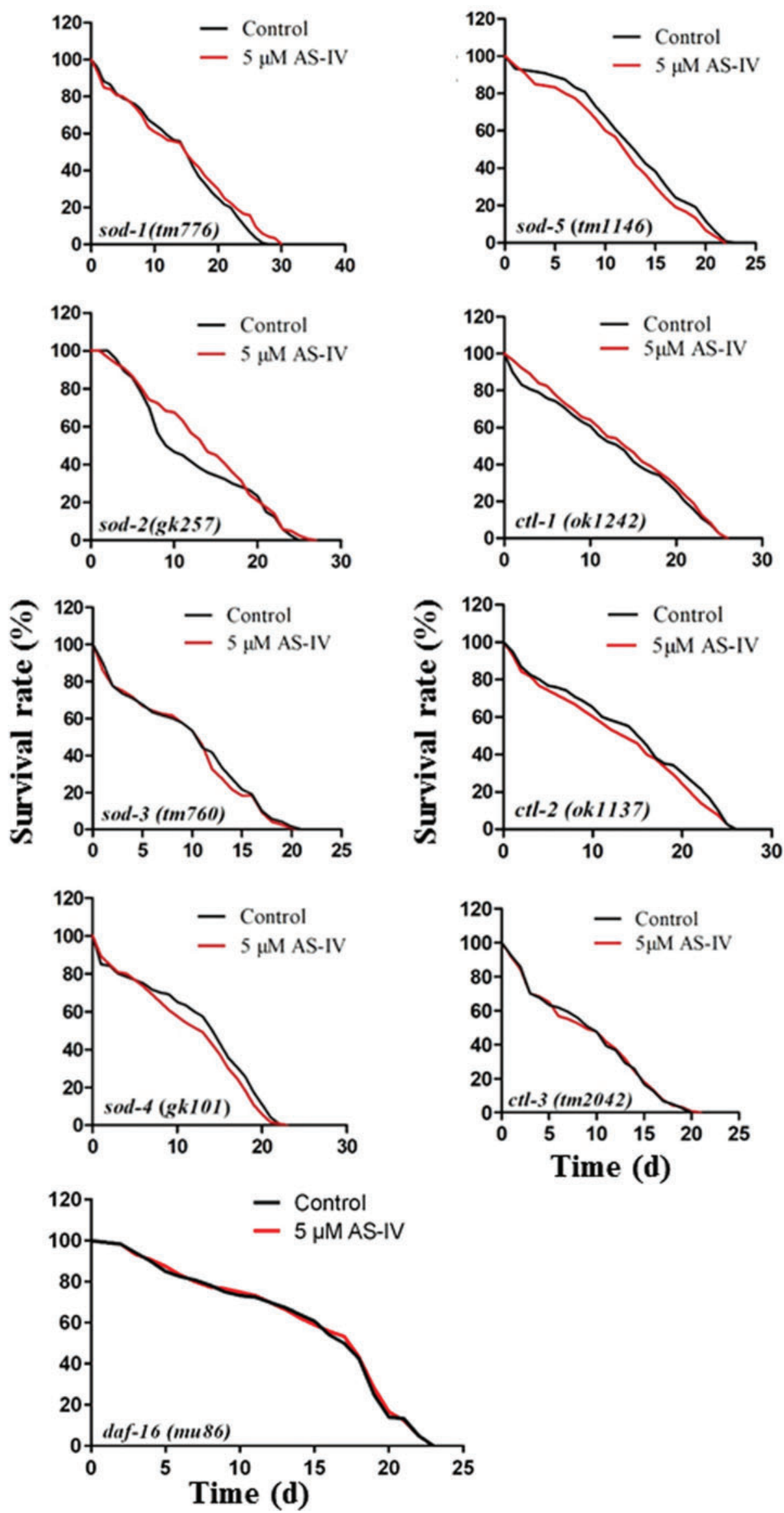

FIG. 7. Effect of AS-IV on the lifespan of nematode mutants. Survival curves were obtained by GraphPad Prism software. The Kaplan-Meier lifespan analysis was performed, and $p$-values were calculated by using log-rank test. Totally, 120 worms were used in each group. Color images are available online. 
aging process. ${ }^{34}$ In our study, the AS-IV group was able to significantly reduce glucose levels. In addition, this study found that succinate levels were significantly elevated in the AS-IV group, suggesting that the antiaging effects of AS-IV may be associated with energy metabolism ${ }^{35,36}$ and mitochondrial function improvement. According to the literature, Sir-2.1 mutant is a commonly used energy metabolism mutant. ${ }^{37}$ To verify whether antiaging of AS-IV is related to energy metabolism of sir-2.1, we conducted experiments by using nematode mutants sir-2.1 (ok434). The results showed that the survival rate of the nematode was significantly increased in the $5 \mu \mathrm{M}$ of the AS-IV group compared with the control group, (Fig. 4). Together, our results suggested that energy metabolism of sir- 2.1 is not the primary mechanism underlying the antiaging effect of AS-IV.

It has been reported that $d a f-16$ can prolong the lifespan of $C$. elegans via shifting glucose metabolism to another type of sugar, trehalose. ${ }^{24}$ In our experiment, we found that AS-IV could not extend the lifespan of nematodes carrying a null mutation of daf-16. The NMR data analysis showed that AS-IV was able to decrease glucose levels. Taken together, the results support the idea that AS-IV may extends lifespan by partially modulating the IIS pathway activity.

(2) Based on the results of ${ }^{1} \mathrm{H}-\mathrm{NMR}$, we found that glutamine and threonine levels were significantly elevated in the aging group $+5 \mu \mathrm{M}$ AS-IV, suggesting that the antiaging effects of AS-IV may be associated with immunomodulation. ${ }^{38}$

The JNK-MAPK signaling pathway is involved in immunomodulatory effects. In $C$. elegans, there are three genes, jnk-1, $k g b-1$, and $k g b-2$ in the JNK-MAPK pathway. $k g b-2$ gene plays a key role in enhancing the immune response toward M9OT and can be regulated by the $j k k-1$ gene during infection. ${ }^{39}$ Studies have shown that jnk-1 plays a biological role under the control of $j k k-1$. JKK-1 is the upstream kinase of JNK-1, and phosphorylation of JNK-1 is required for lifespan extension. ${ }^{40}$ Here, we detected the mRNA expression levels of immunoregulatory genes, $j k k-1$, $j n k-1$, and $k g b-2$. As shown in Figure 5, the transcription of $j k k-1, j n k-1$, and $k g b-2$ was not significantly different in the $5 \mu \mathrm{M}$ of the AS-IV group compared with the control group. Therefore, the results of these experiments suggested that immune regulation is not the key mechanism underlying the antiaging effect of AS-IV.

(3) ${ }^{1} \mathrm{H}-\mathrm{NMR}$ data indicated that the content of glutamate in the aging groups decreased compared with controls, and the content of glutamate in the $5 \mu \mathrm{M}$ of AS-IV group was raised. In general, the lack of glutamate can directly or indirectly reduce antioxidant capacity. ${ }^{41}$ Therefore, we suggest that the antiaging effects of AS-IV may be related to antioxidation.

Oxidative stress is defined as the increased generation of ROS and its oxidative products of biomacromolecules. ${ }^{42}$ It has been shown to play an important role during the process of aging and aging-related degenerative diseases. ${ }^{43,44}$ In addition, free radical-initiated reactions in the body may have a wide variety of detrimental effects on human health. Under normal conditions, the body's ROS are in dynamic equilibrium. However, when the living environment of the organism changes, the body will produce a certain stress reaction and generate a large amount of active oxygen. In the organism, ROS-scavenging enzymes of superoxide dismutase (SOD) and catalase (CAT) show the strong power to maintain the balance of ROS. ${ }^{45}$ SOD removes excess superoxide anion from cells. CAT allows hydrogen peroxide to form water and oxygen molecules. An increase in the activity of these two enzymes indicates an increase in the antioxidant capacity of $C$. elegans.

Aspirin can extend the longevity of $C$. elegans by reducing endogenous levels of ROS and upregulating antioxidant genes (especially sod-3 and ctl-2). ${ }^{29}$ Panax notoginseng polysaccharides significantly extended the lifespan of C.elegans, which might be contributed by the improvement of SOD and $\mathrm{CAT}^{46}$ Baicalein reduces ROS accumulation and prolongs the lifespan of C. elegans. ${ }^{47}$ These studies demonstrated that many compounds can extend the lifespan of nematodes via increasing the activity of antioxidant enzymes and reducing the accumulation of ROS in vivo.

Based on literature listed earlier and the results of ${ }^{1} \mathrm{H}-\mathrm{NMR}$, we hypothesize that the antiaging effect of AS-IV is mainly due to the improvement of antioxidant activity. Accordingly, experiments were designed to test this hypothesis. The results of the antioxidant enzyme assay showed that the activity of SOD and CAT was significantly increased in the $5 \mu \mathrm{M}$ of the AS-IV group compared with the control group (Fig. 6A). Thus, AS-IV can induce the enzyme activity of SOD and CAT. We further tested the response of each gene to AS-IV to determine which key antioxidant genes were involved in the antiaging effect of AS-IV in C. elegans. As shown in Figure 6B and Figure 6C, the transcription levels of sod-3, sod-4, sod-5, ctl-2, and ctl-3 in the $5 \mu \mathrm{M}$ of the AS-IV group were significantly higher than those in the control group $(p<0.05)$. To further determine which antioxidation-related genes regulate the lifespan of nematodes, we investigated the effect of $5 \mu \mathrm{M}$ AS-IV on the lifespan of sod and $c t l$ nematode mutants. The results showed that the lifespan of sod-1 (tm776), sod-2 (gk257), sod-3 (tm760), sod-4 (gk101), and sod-5 (tm1146) mutants and ctl-1 (ok1242), ctl-2 (ok1137), and ctl-3 (ok2042) mutants was not significantly different $(p \geq 0.05)$ in the $5 \mu \mathrm{M}$ of the AS-IV group compared with the control group (Fig. 7).

Generally speaking, hormesis refers to the ability of an organism to react to a compound at low doses, but to inhibit the same agent at high doses. ${ }^{48,49}$ In this study, $5 \mu \mathrm{M}$ of AS-IV could significantly extend the lifespan of $C$. elegans. However, a high concentration of AS-IV can shorten the lifespan of C. elegans (Fig. 1 and Supplementary Fig. S2A). These results demonstrated that there is a hormetic phenomenon in the effect of AS-IV on the organism. This situation might encourage organisms to adapt to the subsequent harmful exposure. Indol- and chromane-type heterocycles had an antiaging effect based on proved beneficial antioxidant effects. Lots of them (e.g., chemically modified flavonoids and introduction of electron-accepting quinonoid substituent into quercetin) showed a hormesis effect at a low concentration. ${ }^{50,51}$ Therefore, the occurrence of the hormetic effect on the nematode aging is speculated to be derived from the action of AS-IV on improving the antioxidant capacity of nematodes. Studies have shown that oligomer $\mathrm{A} \beta_{1-42}$ exerts neurotoxicity and can cause ROS accumulation. ${ }^{52}$ Oxidative stress is suggested to be involved in the pathogenesis of Parkinson's disease. ${ }^{53}$ AS-IV will be a potent alternative therapeutic agent for the treatment of aging-related disease. 


\section{Conclusion}

In summary, for the first time, our data demonstrated that AS-IV can extend the lifespan of $C$. elegans by improving age-related functional declines, inhibiting oxidant stress through upregulation of antioxidant-associated genes, the enhancement of antioxidant activity, and partially by modulating the IIS pathway activity. Thus, these results provide novel insights into the use of AS-IV for the prevention and treatment of aging.

\section{Acknowledgments}

The authors are grateful to doctoral candidate Anastasia M. W. Cooper at the Department of Entomology, Kansas State University, Manhattan, Kansas, USA, and Soheila Fatehi at the Department of Plant Protection, Faculty of Agriculture, University of Tabriz, Tabriz 4560, Islamic Republic of Iran for a critical reading of the article.

\section{Author Disclosure Statement}

No competing financial interests exist.

\section{Funding Information}

This study is funded by the Applied Basic Research of Shanxi Province (No. 201601D202058), 2016 Provincial Support National Research Foundation of Shanxi Province (No. 226546001), Key Laboratory of Effective Substances Research and Utilization in TCM of Shanxi province (201605D111004).

\section{Supplementary Material}

Supplementary Figure S1

Supplementary Figure S2

Supplementary Figure S3

Supplementary Figure S4

Supplementary Figure S5

Supplementary Figure S6

Supplementary Table S1

Supplementary Table S2

\section{References}

1. Mazucanti CH, Cabral-Costa JV, Vasconcelos AR, Andreotti DZ, Scavone C, Kawamoto EM. Longevity pathways (mTOR, SIRT, Insulin/IGF-1) as key modulatory targets on aging and neurodegeneration. Curr Top Med Chem 2015;15:2116-2138.

2. Niccoli T, Partridge L. Ageing as a risk factor for disease. Curr Biol 2012;22:R741-R752.

3. Evason K, Collins JJ, Huang C, Hughes S, Kornfeld K. Valproic acid extends Caenorhabditis elegans lifespan. Aging Cell 2008;7:305-317.

4. Fadini GP, Ceolotto G, Pagnin E, de Kreutzenberg S, Avogaro A. At the crossroads of longevity and metabolism: The metabolic syndrome and lifespan determinant pathways. Aging Cell 2011;10:10-17.

5. Selman C, Partridge L, Withers DJ. Replication of extended lifespan phenotype in mice with deletion of insulin receptor substrate 1. PLoS One 2011;6:e16144.

6. Tepper RG, Ashraf J, Kaletsky R, Kleemann G, Murphy CT, Bussemaker HJ. PQM-1 complements DAF-16 as a key transcriptional regulator of DAF-2-mediated development and longevity. Cell 2013;154:676-690.

7. Mishur RJ, Khan M, Munkácsy E, et al. Mitochondrial metabolites extend lifespan. Aging Cell 2010;15:336-348.

8. Li J, Cui XD, Wang ZH, Li YY. rBTI extends Caenorhabditis elegans lifespan by mimicking calorie restriction. Exp Gerontol 2015;67:62-71.

9. Kenyon CJ. The genetics of ageing. Nature 2010;464:504 512.

10. Tullet JM, Hertweck M, An JH, et al. Direct inhibition of the longevity promoting factor SKN-1 by insulin-like signaling in C. elegans. Cell 2008;132:1025-1038.

11. Zheng SQ, Huang XB, Xing TK, Ding AJ, Wu GS, Luo HR. Chlorogenic Acid Extends the Lifespan of Caenorhabditis elegans via Insulin/IGF-1 Signaling Pathway. J Gerontol A Biol Sci Med Sci 2017;72:464-472.

12. Han L, Chen KJ. Advances in experimental pharmacological studies of effects of astragalus on cardiovascular system. Chin J Integr Med 2001;7:146-151.

13. Gui DK, Huang JH, Guo YP, et al. Astragaloside IV ameliorates renal injury in streptozotocin-induced diabetic rats through inhibiting NF- $\kappa \mathrm{B}$-mediated inflammatory genes expression. Cytokine 2013;61:970-977.

14. Gui DK, Guo YP, Wang F, et al. Astragaloside IV, a novel antioxidant, prevents glucose-induced podocyte apoptosis in vitro and in vivo. PLoS One 2012;7:e39824.

15. Wang $X$, Zhang A, Han Y, et al. Urine metabolomics analysis for biomarker discovery and detection of jaundice syndrome in patients with liver disease. Mol Cell Proteomics 2012;11:370-380.

16. Heude C, Nath J, Carrigan JB, Ludwig C. Nuclear magnetic resonance strategies for metabolic analysis. Adv Exp Med Biol 2017;965:45-76.

17. Chun L, Gong JK, Yuan FL, et al. Metabotropic GABA signalling modulates longevity in C.elegans. Nat Commun 2015;6:8828.

18. Dhawan R, Dusenbery DB, Williams PL. A comparison of metal-induced lethality and behavioral responses in the nematode Caenorhabditis elegans. Environ Toxicol Chem 2000;19:3061-3067.

19. Geier FM, Want EJ, Leroi AM, Bundy JG. Cross-platform comparison of Caenorhabditis elegans tissue extraction strategies for comprehensive metabolome coverage. Anal Chem 2011;83:3730-3736.

20. Zhang J, Xue X, Yang Y, Ma W, Han Y, Qin X. Multiple biological defects caused by calycosin-7-O- $\beta$-D-glucoside in the nematode Caenorhabditis elegans are associated with the activation of oxidative damage. J Appl Toxicol 2018;38:801-809.

21. Lee SJ, Hwang AB, Kenyon C. Inhibition of respiration extends $C$. elegans life span via reactive oxygen species that increase HIF-1 activity. Curr Biol 2010;20:2131-2136.

22. Pfaffl MW. A new mathematical model for relative quantification in real-time RT-PCR. Nucleic Acids Res 2001;29:e45.

23. Li AP, Li ZY, Sun HF, Li K, Qin XM, Du GH. Comparison of two different astragali radix by a ${ }^{1} \mathrm{H}-\mathrm{NMR}$ based metabolomic approach. J Proteome Res 2015;14:2005-2016.

24. Hibshman JD, Doan AE, Moore BT, et al. daf-16/FoxO promotes gluconeogenesis and trehalose synthesis during starvation to support survival. eLife 2017;6:e30057.

25. Lin $\mathrm{K}$, Hsin H, Libina N, Kenyon C. Regulation of the Caenorhabditis elegans longevity protein DAF-16 by insulin / IGF-1 and germline signaling. Nat Genet 2001;28: 139-145. 
26. Chen P, Xie YQ, Shen E, et al. Astragaloside IV attenuates myocardial fibrosis by inhibiting TGF- $\beta 1$ signaling in coxsackievirus B3-induced cardiomyopathy. Eur J Pharmacol 2011;658:168-174.

27. Chan WS, Durairajan SSK, Lu JH, et al. Neuroprotective effects of Astragaloside IV in 6-hydroxydopamine-treated primary nigral cell culture. Neurochem Int 2009;55:414422.

28. Zhang NN, Li ZC, Mu W, et al. Calorie restrictioninduced SIRT6 activation delays aging by suppressing NF- $\kappa \mathrm{B}$ signaling. Cell Cycle 2016;15:1009-1018.

29. Ayyadevara S, Bharill P, Dandapat A, et al. Aspirin inhibits oxidant stress, reduces age-associated functional declines, and extends lifespan of Caenorhabditis elegans. Antioxid Redox Signal 2013;18:481-490.

30. Bass TM, Weinkove D, Houthoofd K, Gems D, Partridge L. Effects of resveratrol on lifespan in Drosophila melanogaster and Caenorhabditis elegans. Mech Ageing Dev 2007; 128:546-552.

31. Kenyon C. A conserved regulatory system for aging. Cell 2001;105:165-168.

32. Morimoto RI. Stress, aging, and neurodegenerative disease. N Engl J Med 2006;355:2254-2255.

33. Shmookler Reis RJ, Xu LL, Lee H, et al. Modulation of lipid biosynthesis contributes to stress resistance and longevity of C. elegans mutants. Aging 2011;3:125-147.

34. Hoffman JM, Soltow QA, Li S, Sidik A, Jones DP, Promislow DE. Effects of age, sex, and genotype on highsensitivity metabolomic profiles in the fruit fly, Drosophila melanogaster. Aging Cell 2014;13:596-604.

35. Xia J, Sinelnikov IV, Han B, Wishart DS. MetaboAnalyst 3.0-making metabolomics more meaningful. Nucleic Acids Res 2015;43:W251.

36. Gong MJ, Han B, Wang SM, Liang SW, Zou ZJ. Icariin reverses corticosterone-induced depression-like behavior, decrease in hippocampal brain-derived neurotrophic factor (BDNF) and metabolic network disturbances revealed by NMR-based metabonomics in rats. J Pharm Biomed Anal 2016;123:63-73.

37. Bamps S, Wirtz J, Savory FR, Lake D, Hope IA. The Caenorhabditis elegans sirtuin gene, sir-2.1, is widely expressed and induced upon caloric restriction. Mech Ageing Dev 2009;130:762-770.

38. Zhang WJ, Frei B. Astragaloside IV inhibits NF- $\kappa \mathrm{B}$ activation and inflammatory gene expression in LPS-treated mice. Mediators Inflamm 2015;274314.

39. Marudhupandiyan S, Balamurugan K. Intrinsic JNK-MAPK pathway involvement requires daf-16-mediated immune response during Shigella flexneri infection in C. elegans. Immunol Res 2016;65:1-13.

40. Oh SW, Mukhopadhyay A, Svrzikapa N, Jiang F, Davis RJ, Tissenbaum HA. JNK regulates lifespan in Caenorhabditis elegans by modulating nuclear translocation of forkhead transcription factor/DAF-16. Proc Natl Acad Sci USA 2005;102:4494-4499.

41. Lu Y, Li S, Wu HF, et al. Beneficial effects of astragaloside IV against angiotensin II-induced mitochondrial dysfunction in rat vascular smooth muscle cells. Int $\mathbf{J}$ Mol Med 2015;36:1223-1232.
42. Li T, Chen S, Feng T, Dong J, Li Y, Li H. Rutin protects against aging-related metabolic dysfunction. Food Funct 2016;7:1147-1154.

43. Ma XK, Guo DD, Peterson EC, Dun Y, Li DY. Structural characterization and anti-aging activity of a novel extracellular polysaccharide from fungus Phellinus sp. in a mammalian system. Food Funct 2016;7:3468-3479.

44. Zhong WQ, Liu N, Xie YG, Zhao YM, Song X, Zhong WM. Antioxidant and anti-aging activities of mycelial polysaccharides from Lepista sordida. Int J Biol Macromol 2013;60:355-359.

45. Halliwell B. Antioxidant defence mechanisms: From the beginning to the end (of the beginning). Free Radic Res 1999;31:261-272.

46. Feng SL, Cheng HR, Xu Z, et al. Thermal stress resistance and aging effects of Panax notoginseng polysaccharides on Caenorhabditis elegans. Int J Biol Macromo 2015;81:188194.

47. Havermann S, Rohrig R, Chovolou Y, Humpf HU, Wätjen W. Molecular effects of baicalein in Hct116 cells and Caenorhabditis elegans: Activation of the Nrf2 signalling pathway and prolongation of lifespan. J Agric Food Chem 2013;61:2158-2164.

48. Lindsay DG. Nutrition, hormetic stress and health. Nutr Res Rev 2005;18:249-258.

49. Hansen SL, Purup S, Christensen LP. Bioactivity of falcarinol and the influence of processing and storage on its content in carrots (Daucus carota L.). J Sci Food Agr 2003; 83:1010-1017.

50. Lucia R, Nataša $M$, Martin $S$, Ivana $M$, Magdaléna $M$, Stefan B. PP39-Indole- and Chromane-type heterocycles: Playing with molecules to defeat ageing. Free Radic Biol Med 2015;86:S32.

51. Sun HY, Zheng M, Song JY, et al. Multiple-species hormetic phenomena induced by indole: A case study on the toxicity of indole to bacteria, algae and human cells. Sci Total Environ 2019;657:46-55.

52. Sun QR, Jia N, Wang WX, Jin H, Xu JH, Hu HT. Protective Effects of Astragaloside IV against Amyloid Beta1-Beta42 neurotoxicity by inhibiting the mitochondrial permeability transition pore opening. PLoS One 2014;9:e98866.

53. Zhang ZG, Wu L, Wang JL, et al. Astragaloside IV prevents $\mathrm{MPP}^{+}$-induced SH-SY5Y cell death via the inhibition of Bax-mediated pathways and ROS production. Mol Cell Biochem 2012;364:209-216.

Address correspondence to: Jianqin Zhang

Modern Research Center for Traditional Chinese Medicine Shanxi University Taiyuan 030006 China

E-mail: jiangqinzh3@sxu.edu.cn

Received: February 5, 2020 Accepted: August 1, 2020 